\title{
Belgeo
}

Revue belge de géographie

$1 \mid 2017$

Miscellaneous

\section{In memoriam Jean-Pierre Vandenbosch (1932-2018)}

\section{(2) OpenEdition}

1 Journals

Édition électronique

URL : https://journals.openedition.org/belgeo/20535

DOI : $10.4000 /$ belgeo.20535

ISSN : 2294-9135

Éditeur :

National Committee of Geography of Belgium, Société Royale Belge de Géographie

\section{Référence électronique}

«In memoriam Jean-Pierre Vandenbosch (1932-2018)», Belgeo [En ligne], 1 | 2017, mis en ligne le 22 janvier 2018, consulté le 28 juin 2022. URL : http://journals.openedition.org/belgeo/20535; DOI : https://doi.org/10.4000/belgeo.20535

Ce document a été généré automatiquement le 29 septembre 2020

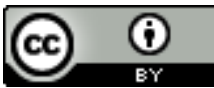

Belgeo est mis à disposition selon les termes de la licence Creative Commons Attribution 4.0 International. 


\section{In memoriam Jean-Pierre Vandenbosch (1932-2018)}

Jean-Pierre Vandenbosch est décédé ce 2 janvier 2018. Licencié et agrégé de l'Université Libre de

Bruxelles (1955), il fit pratiquement toute sa carrière d'enseignant à «feu » l'Athénée Royal Jules Bordet, à Bruxelles. Homme de réflexion et d'action, il s'impliqua beaucoup dans la FEGEPRO (Fédération des Professeurs de Géographie), dont il fut le principal animateur pendant près de 30 ans et aussi le président de 1991 à 1993. Dès les années 1970, il chercha à renouveler non seulement les productions pédagogiques mais encore la didactique de l'enseignement de la discipline au cœur de laquelle il proposait d'utiliser pas à pas la géographie pour mener l'adolescent vers son épanouissement. Il initia de nombreux projets au niveau national et international, notamment dans le cadre de la

Conférence permanente européenne des Associations de Professeurs de Géographie, dont il fut le secrétaire. Convaincu de l'utilité de la géographie comme outil fondamental

d'appropriation, de compréhension et de connaissance de la réalité à la fois naturelle et sociale qui nous entoure, il fut un grand

défenseur d'une formation géographique de qualité pour tous les êtres humains.

Sans aucun doute, nous avons donc perdu à la fois un grand géographe, un grand enseignant et un grand humaniste.

Vous trouverez ci-dessous un texte qu'il avait 
écrit en 2009 et qui nous éclaire sur sa vision de la géographie.

Bernadette Mérenne-Schoumaker

\section{Aujourd'hui, gagner c'est gérer l'incertitude}

\section{Jean-Pierre Vandenbosch}

1 Licencié-agrégé en sciences géographiques

Professeur honoraire de l'enseignement secondaire

Ancien animateur et ancien président de la FEGEPRO

2 Formé à l'Université Libre de Bruxelles dans le début des années 1950 par Pierre Gourou, un maître de la géographie française, mon enseignement dans le secondaire a très vite été confronté aux réalités d'un monde en pleine mutation.

De lectures et d'excursions géographiques en voyages sur d'autres continents, de lectures d'ouvrages pédagogiques et psychologiques en expérimentations en classe, de l'observation attentive de l'actualité (1968 !) à la collaboration assidue avec le Bureau d'Information des Communautés européennes pour la Belgique, mon enseignement s'est imprégné de toutes les informations recueillies au cours de ces lectures et de ces déplacements.

4 En particulier, le livre d'Yves Lacoste La géographie, ça sert d'abord à faire la guerre a marqué un tournant dans ma manière d'enseigner ainsi qu'un peu plus tard la pédagogie du contrat et $\mathrm{du}$ projet qui allait risquer de me mettre physiquement à genoux.

5 C'est alors, dans les années 1970, que l'Inspecteur Julien Vereerstraeten m’a suggéré d'entrer dans le Conseil d'Administration de la FEGEPRO, Fédération des Géographes professeurs, société bilingue à l'époque, au sein de laquelle j'ai fait la connaissance d'Emile Mérenne et où des liens étroits ont été tissés avec la Société Géographique de Liège grâce à Bernadette Mérenne.

6 Afin de donner un cadre institutionnel à notre désir d'innovation, il fut créé, au sein de la FEGEPRO, la Commission exécutive francophone, dont le secrétariat fut assuré par Jacques Pauwels et dont j'assurai l'animation. Devant le besoin criant de documents pédagogiques, la Commission exécutive lança Les Feuillets d'Informations, toujours édités grâce à Bernard Andries et aux membres du Conseil d'Administration de la FEGEPRO, aujourd'hui uniquement francophone. Cette Commission entreprit également avec l'appui du Bureau de Bruxelles des Communautés européennes la publication des «dossiers bleus». Dans la rédaction de ces documents, l'apport dynamique de Bernadette Mérenne fut important.

7 Enfin, tout ce remue-méninge demandait à être structuré, ce qui fut entrepris par un groupe de géographes enseignant dans divers réseaux, à différents niveaux et dans les types d'enseignement de l'époque, réunis à Sart-lez-Spa en 1976. Il en résulta une brochure, La Géographie, un Outil de Formation, appelé GOF dans notre jargon, et qui introduisait la démarche informatique dans nos raisonnements.

8 J'assumai également le secrétariat de la Conférence permanente européenne des Associations de Professeurs de Géographie. L'édition tous les deux ans de la brochure 
EUROGEO par les soins du collègue néerlandais Henk Meijer mit en valeur les difficultés de l'approche géographique en Europe.

9 En parallèle, je participais à la constitution de documents pédagogiques qui concernaient la géographie de l'Agglomération de Bruxelles pour la revue L'École et la Ville.

10 Ce travail à différentes échelles spatiales fut particulièrement fécond. Il m'amena à relativiser de nombreuses notions et à me convaincre que la géographie, par la gymnastique du changement d'échelle, constituait un apport essentiel dans la formation des jeunes.

11 L'introduction des ordinateurs dans les écoles au cours des années 1980 a été un nouveau défi à relever. Je crains qu'il soit très difficile de s'affranchir des méthodes traditionnelles d'enseignement et d'innover en utilisant l'outil informatique. C'est pourquoi, je crois que dans un monde en évolution permanente, tant du point de vue technologique que social et éthique, l'école qui n'est plus portée par un environnement motivant et qui n'est plus la seule source du savoir, se doit de réfléchir à ses objectifs.

«L'éducatique» (A. Ducrocq, 1981) pourrait être une voie de réflexion. Par l'usage judicieux des moyens techniques, elle devrait permettre d'obtenir de meilleurs résultats dans l'enseignement. Dès lors, trois questions se poseraient aux spécialistes de chaque discipline :

1. Quel est l'apport spécifique d'une discipline à la formation des individus?

2. Quelles sont les matières de cette discipline qui se prêtent le mieux à faire acquérir cet apport spécifique?

3. Quelles sont les techniques d'enseignement à mettre en œuvre pour faire acquérir cet apport spécifique avec le plus d'efficacité au plus grand nombre d'individus?

Appliquée à la géographie, les réponses aux trois questions pourraient être :

1. La géographie participe à la formation des individus ;

* par des démarches d'analyse scientifique;

* par des démarches méthodologiques partant de l'espace vécu et perçu complété par l'espace donné et raisonné pour aboutir à un espace intégré et volontaire ;

* par l'application de grilles d'analyse à des faits d'actualité mettant en évidence les niveaux spatiaux d'action ;

* par la détermination d'ensembles spatiaux par superposition de cartes à la même échelle ;

* par la gymnastique du changement d'échelle permettant de mettre en évidence la nature différente des problèmes suivant leur échelle spatiale ;

* par la gymnastique du changement de références en utilisant :

- des projections cartographiques différentes, si importantes dans l'approche stratégique des problèmes mondiaux,

- des regroupements scientifiques mais variés de séries statistiques donnant des images différentes d'une même réalité.

15 2. Les démarches géographiques peuvent être appliquées à n'importe quelle situation instable exigeant une décision. Le choix de cette situation dépendra de l'intérêt et du niveau des élèves ainsi que des documents disponibles et des impératifs institutionnels. Dès lors se posera la question : "parmi les nombreux documents rassemblés, lesquels choisir pour agir efficacement?». 


\section{Monde du
Dossier).}

«Dans l'école, à l'heure du numérique, l'enseignant change de statut. Il n'a plus le monopole de l'introduction du savoir et des documents dans la classe mais devient celui qui valide les recherches, aide à repérer les sources dignes de confiance, développe l'esprit critique d'élèves qui travaillent en groupes [...].

Aujourd'hui, on constate que, si les pratiques pédagogiques liées au numérique existent [...], elles restent largement minoritaires. Le monde universitaire s'en est saisi pour développer l'enseignement à distance mais il s'agit souvent d'une mise en ligne de cours conçus pour l'enseignement traditionnel et qui n'exploitent qu'à la marge les possibilités du numérique et de l'interactivité [...].

Manque de formation des enseignants, peur de se trouver sur un terrain que les élèves connaissent mieux qu'eux par leurs activités de loisirs, difficulté bien compréhensible à remettre en cause une pratique professionnelle de deux siècles, ...».

21 A la page 7 du même dossier sous le titre "Internet et pédagogie ", Luc Trouche, directeur d'EducTice à l'Institut national de recherche pédagogique (INRP) écrit :

"A propos des usages pédagogiques d'Internet et de l'environnement numérique, le développement des associations d'enseignants concepteurs et "partageurs " de ressources est un fait majeur. [...] La conception et l'usage de nouvelles activités supposent de repenser l'organisation globale de l'école et de l'enseignement. [...] En ce sens, le développement d'associations d'enseignants en ligne est décisif pour les évolutions à venir.»

Il reste beaucoup à faire pour que s'installe un consensus sur l'application de l'éducatique à la géographie et pour que des documents de qualité, mis à jour en permanence et indispensables à cette application, soient réalisés. Mais, heureusement, la FEGEPRO existe et son dynamisme permet tous les espoirs. 